\title{
Treinamento muscular inspiratório em crianças com leucemia aguda: resultados preliminares
}

\author{
Inspiratory muscle training in patients with acute leukemia: preliminary results
}

\begin{abstract}
Thalita Medeiros F. de Macedo ${ }^{1}$, Kátia Myllene C. Oliveira², Janiara Borges da C. Melo², Mariana Germano de Medeiros², Wilson C. de Medeiros Filho ${ }^{3}$, Gardênia Maria H. Ferreira', Raíssa de Oliveira Borja ${ }^{1}$, Tania Fernandes Campos ${ }^{5}$,

Karla Morganna P. P. de Mendonça ${ }^{4}$
\end{abstract}

\section{RESUMO}

Objetivo: Avaliar o efeito do treinamento muscular inspiratório em crianças com leucemia aguda.

Métodos: Trata-se de um estudo quase experimental com grupo controle. Quatorze pacientes com diagnóstico de leucemia aguda e idade entre cinco e 14 anos foram submetidos à avaliação da mobilidade torácica e da força muscular respiratória e divididos em dois grupos (A e B). Os pacientes do grupo A realizaram treinamento muscular inspiratório domiciliar por 15 minutos, duas vezes por dia, durante dez semanas, através do aparelho Threshold ${ }^{\circledR}$, com carga de $30 \%$ da pressão inspiratória máxima, reajustada após reavaliações semanais. O grupo B (controle) realizou uma avaliação das pressões respiratórias máximas inicialmente e após dez semanas. Os dados foram analisados com o SPSS 15.0. Aplicou-se o teste $t$ para analisar as diferenças entre as pressões inspiratória máxima e expiratória máxima entre os dois grupos e o teste de correlação de Pearson para analisar associações das medidas de pressão inspiratória e pressão expiratória com as diferentes cargas utilizadas.

Resultados: Constatou-se ganho significativo de $35 \%$ nas pressões inspiratória máxima e expiratória máxima no grupo A ao término do treinamento. Houve correlação positiva entre os níveis de carga utilizada e a pressão inspiratória máxima $(p<0,0001)$ e a pressão expiratória máxima $(p=0,0001)$.
Conclusões: $\mathrm{O}$ treinamento muscular inspiratório pode ser eficaz no ganho de força muscular em crianças em tratamento de leucemia aguda.

Palavras-chave: leucemia; exercícios respiratórios; músculos respiratórios.

\section{ABSTRACT}

Objective: To evaluate the effect of inspiratory muscle training in children with acute leukemia.

Methods: This is a quasi-experimental study with a control group. Fourteen patients with 5-14 years old and a diagnosis of acute leukemia were evaluated regarding their thoracic mobility and respiratory muscle strength. They were divided in two groups (A and B). Group A received domiciliary inspiratory muscle training during 15 minutes, twice a day, for ten weeks, with a Threshold ${ }^{\circledR}$ device using a load of $30 \%$ of the maximal inspiratory pressure, readjusted after weekly evaluations. Group B patients were tested regarding their maximum respiratory pressures in their first evaluation and after ten weeks. Student $t$-test was used to evaluate maximum inspiratory and expiratory pressures between groups Pearson's correlation test examined the association of inspiratory and expiratory pressures with the different loads, using SPSS 15.0 software.
Instituição: Universidade Federal do Rio Grande do Norte (UFRN), Natal, RN, Brasil

${ }^{1}$ Mestranda do Programa de Pós-Graduação em Fisioterapia da UFRN; Fisioterapeuta Graduada pela UFRN, Natal, RN, Brasil

${ }^{2}$ Fisioterapeuta Graduada pela UFRN, Natal, RN, Brasil

${ }^{3}$ Onco-Hematologista Pediátrico do Hospital Infantil Varela Santiago, Natal, RN, Brasil

${ }^{4}$ Doutora em Ciências da Saúde pela UFRN; Professora Adjunta do Curso de Fisioterapia da UFRN, Natal, RN, Brasil

${ }^{5}$ Doutora em Psicobiologia pela UFRN; Professora Adjunta do Curso de Fisioterapia da UFRN, Natal, RN, Brasil
Endereço para correspondência:

Karla Morganna P. P. de Mendonça

Avenida Ayrton Senna, 880 - Condomínio Bosque das Palmeiras, casa 82 Cidade dos Bosques - Nova Parnamirim

CEP 59151-600 - Parnamirim/RN

E-mail: kmorganna@ufrnet.br

Fonte financiadora: Fundação de Apoio à Pesquisa do Rio Grande do Norte convênio n 299966/2008

Conflitos de interesse: nada a declarar

Recebido em: 25/9/2009

Aprovado em: 15/4/2010 
Results: A significant improvement of $35 \%$ was observed in the maximum inspiratory and expiratory pressures in Group $A$ at the end of the training. There was a strong positive correlation between the used load levels and maximal inspiratory $(p<0.0001)$ and expiratory $(p=0.0001)$ pressures.

Conclusions: Inspiratory muscle training can be effective for improving inspiratory muscle strength in children beeing treated for acute leukemia.

Key-words: leukemia; breathing exercises; respiratory muscles.

\section{Introdução}

Toda neoplasia maligna que acomete indivíduos menores de 15 anos é considerada câncer infantil ${ }^{(1)}$. O câncer pediátrico representa de 0,5 a $3 \%$ de todos os tumores da população(2), sendo a leucemia o tipo mais comum ${ }^{(1)}$. No Brasil sua incidência é análoga à encontrada nos demais países, variando de 25 a $35 \%$ das neoplasias pediátricas ${ }^{(3)}$.

O metabolismo de pacientes com câncer sofre modificações drásticas devido ao estresse criado pela própria doença, como também pelos efeitos colaterais produzidos pelos tratamentos tradicionais administrados (cirurgia, quimioterapia ou radiação). Tais modificações metabólicas podem se associar à depressão psicológica e à diminuição no apetite, fatores que levam os pacientes a iniciar um ciclo vicioso de perda de massa muscular e redução nos níveis de atividade física, resultando em um estado de fraqueza generalizada ${ }^{(4)}$. Além disso, a quimioterapia pode causar danos à medula óssea e, dessa forma, prejudicar a produção de glóbulos vermelhos, com anemia e diminuição na capacidade de transporte de oxigênio no sangue e, portanto, redução do fornecimento deste às células ${ }^{(5)}$. Estudos têm demonstrado uma relação entre anemia e fadiga, no entanto, o mecanismo pelo qual ocorre essa relação é desconhecido em pacientes com câncer ${ }^{(6)}$. A fadiga é um sintoma relatado por $95 \%$ dos pacientes em tratamento com quimioterapia ou radioterapia ${ }^{(7)}$. De acordo com Curt $e t a^{(8)}$, a fadiga pode prejudicar o desempenho das atividades de vida diária (AVD) e levar a efeitos negativos na qualidade de vida ${ }^{(4,8,9)}$.

As limitações nas AVD favorecem a manutenção da inatividade e a falta de condicionamento físico, podendo afetar vários órgãos e sistemas, incluindo o respiratório, o que facilita distúrbios na ventilação ${ }^{(10)}$. Como consequência, pode ocorrer diminuição da força ou resistência muscular e surgir de forma prematura a fadiga dos músculos ventilatórios ${ }^{(11)}$.
$\mathrm{Na}$ tentativa de buscar alternativas para minimizar os efeitos na fraqueza e/ou fadiga dos músculos respiratórios, em 1976 foi realizado o primeiro estudo com o propósito de avaliar as repercussões do treinamento dos músculos inspiratórios em indivíduos saudáveis ${ }^{(12)}$. Desde então, o número de estudos a respeito do treinamento muscular inspiratório (TMI) tem aumentado, sendo utilizado no processo de reabilitação de diversas doenças cujo parâmetro comum é a fraqueza e/ou a fadiga da musculatura respiratória ${ }^{(13-19)}$. $\mathrm{Na}$ maioria dos estudos, esse treinamento se mostra eficaz no ganho de força e na resistência da musculatura respiratória, levando à redução da dispneia, melhora no desempenho de AVD e redução de complicações pulmonares pós-operatórias.

Considerando que a fraqueza e a fadiga muscular podem ser observadas em pacientes com câncer em decorrência da quimioterapia e da imobilidade e, ainda, a inexistência de pesquisas que investiguem as consequências da quimioterapia nos músculos respiratórios dessa população, o presente estudo se propôs a avaliar o efeito do treinamento muscular inspiratório em crianças com leucemia aguda na fase de manutenção do tratamento quimioterápico.

\section{Método}

Este estudo foi aprovado pelo Comitê de Ética e Pesquisa da Universidade Federal do Rio Grande do Norte, de acordo com a resolução 196/1996 do Conselho Nacional de Saúde e consistiu em uma pesquisa quase experimental com grupo controle.

Foi utilizada uma amostra de conveniência composta por todas as crianças com idade entre cinco e 17 anos que apresentavam diagnóstico de leucemia aguda e que se encontravam na fase de manutenção da quimioterapia em ambulatório de Onco-Hematologia de um hospital de referência no Estado do Rio Grande do Norte. Não foram elegíveis as crianças com diagnóstico de doença pulmonar crônica, doença neuromuscular, aquelas que haviam realizado ou estivessem em tratamento radioterápico e as que apresentassem comprometimento neurológico.

No período compreendido entre agosto de 2008 e maio de 2009, 18 crianças compareceram ao ambulatório do referido hospital. Os pais e/ou responsáveis destas foram esclarecidos acerca dos objetivos e da metodologia proposta para o estudo e, posteriormente, todos assinaram o termo de consentimento livre e esclarecido.

É importante ressaltar que, mesmo após o consentimento dos seus pais e/ou responsáveis, as crianças apenas 
participariam do estudo se estivessem de acordo. Estas também foram esclarecidas sobre a pesquisa em linguagem acessível à faixa etária e por meio de cartilha desenvolvida para o estudo. Após os esclarecimentos e consentimento, todas as crianças foram recrutadas e avaliadas. As crianças foram sorteadas e distribuídas aleatoriamente em dois grupos, com nove sujeitos em cada, denominados grupos A e B. Todos os pacientes, em ambos os grupos, realizaram avaliação respiratória no instante inicial, avaliação das pressões respiratórias máximas após dez semanas, acompanhamento clínico durante todo o período pelo médico responsável e receberam a terapia medicamentosa de rotina para a fase de manutenção da quimioterapia. O grupo A realizou também o treinamento muscular inspiratório domiciliar durante dez semanas, com avaliação das pressões respiratórias máximas ao término de cada semana.

Todos os pacientes realizaram a avaliação inicial, que incluiu coleta de informações referentes aos dados pessoais, tipo de leucemia, médico responsável, cirtometria torácica e pressões respiratórias máximas. A fadiga e a dispneia foram acompanhadas pela escala visual analógica (EVA) de Borg modificada ${ }^{(20)}$. A saturação periférica de oxigênio e a frequência cardíaca foram monitoradas durante as avaliações.

A avaliação da mobilidade torácica foi realizada a partir da cirtometria. Com uma fita métrica de $150 \mathrm{~cm}$ posicionada inicialmente na linha axilar, a criança foi orientada, com um comando verbal, a realizar uma expiração máxima (E1), seguida de uma inspiração máxima (I), finalizando com uma nova expiração máxima (E2). O mesmo procedimento foi aplicado com a fita métrica posicionada no apêndice xifoide. Os valores referentes à expansão torácica foram obtidos pela equação I - E1, enquanto aqueles referentes à retração pela equação I - E2 ${ }^{(21)}$.

A avaliação da força muscular respiratória foi obtida por medida da pressão inspiratória máxima (PImáx) e da pressão expiratória máxima (PEmáx), de acordo com o método proposto por Black e Hyatt ${ }^{(2)}$, utilizando um manovacuômetro digital (Globalmed - MVD 300), calibrado de -300 $\mathrm{a}+300 \mathrm{cmH}_{2} \mathrm{O}$, sensível à variação de cada centímetro de água. Acoplado a este, foi utilizado um bocal com orifício de aproximadamente $1 \mathrm{~mm}$ de diâmetro na parte superior para dissipar pressões adicionais causadas pelos músculos faciais e da orofaringe ${ }^{(23)}$. Para evitar escape de ar, foi aplicado um clipe nasal e o paciente foi orientado a realizar um ajuste adequado dos lábios ao bocal. Para medir a PImáx, o paciente foi orientado a realizar uma inspiração máxima, a partir do volume residual e, para a PEmáx, orientou-se efetuar uma expiração máxima a partir da capacidade pulmonar total. Para minimizar o uso da musculatura acessória da face, o avaliador fez contenção manual das bochechas durante a avaliação da PEmáx. As pressões medidas foram sustentadas por pelo menos um segundo, sendo efetuadas três manobras de PImáx e PEmáx e considerada a de maior valor. Ofereceu-se um minuto de descanso entre cada manobra e, por ser um teste esforço-dependente, foi fornecido encorajamento verbal para sua realização. Durante todo o teste, o paciente deveria estar sentado confortavelmente.

Os pacientes do Grupo A realizaram a avaliação das pressões respiratórias máximas quando selecionados e ao final de cada semana de treinamento, totalizando 11 medições, todas por um mesmo avaliador e no mesmo turno, no ambulatório do hospital. O Grupo B realizou a manovacuometria durante a avaliação inicial e após dez semanas.

Para o treinamento muscular inspiratório, foi utilizado o aparelho Threshold ${ }^{\circledR}$ IMT (Respironics Inc., Cedar Grove, New Jersey, USA), composto por um corpo plástico cilíndrico, um bocal sem furo, e uma válvula unidirecional controlada por uma mola, oferecendo uma variação de carga de 7 a $41 \mathrm{cmH}_{2} \mathrm{O}$, com ajuste de pressão de $2 \mathrm{em} 2 \mathrm{cmH}_{2} \mathrm{O}$. A carga pressórica imposta pelo aparelho foi de $30 \%$ da PImáx durante todo o treinamento. A carga da primeira semana foi ajustada pela PImáx obtida na avaliação respiratória inicial. Essa carga foi ajustada de acordo com a PImáx obtida na avaliação realizada ao término de cada semana de treinamento.

Inicialmente, todas as crianças foram ensinadas a utilizar o aparelho Threshold ${ }^{\circledR}$ IMT e passaram por um momento de adaptação, utilizando o aparelho com carga mínima. Os pacientes realizaram o treinamento muscular inspiratório duas vezes por dia (uma no turno matutino e outra no vespertino, aproximadamente no mesmo horário e respeitando um intervalo mínimo de uma hora após as refeições), todos os dias da semana, durante dez semanas, totalizando 140 sessões. A cada semana, o mesmo pesquisador realizou uma nova reavaliação das pressões respiratórias máximas, ajustou a carga pressórica, sempre com o critério de 30\% da PImáx. Esse pesquisador supervisionava uma sessão de treinamento, após cada reajuste da carga, para certificar-se de que estava sendo realizado corretamente.

As crianças realizaram o treinamento sentadas confortavelmente, utilizando um clipe nasal, com a boca acoplada ao bocal do aparelho e respiravam de forma normal por 15 minutos, com intervalo para descanso de um minuto a cada 
dez respirações. Seus pais também preencheram um diário informando a hora em que o treinamento foi realizado e por quantos minutos, em cada dia da semana. Ainda poderia ser informada a ocorrência de qualquer limitação durante o treinamento. A cada reavaliação semanal da força muscular respiratória, as anotações do diário eram checadas pelo pesquisador responsável.

A análise dos dados foi realizada pelo programa Statistical Package for the Social Science (SPSS 15.0). Inicialmente aplicouse a estatística descritiva para caracterizar a amostra. Após verificação de normalidade, foi utilizado o teste $t$ de Student pareado para buscar diferenças entre a PImáx e PEmáx antes e no final do TMI no Grupo A, bem como diferentes comparações entre a medida da PImáx e PEmáx antes do TMI com as dez medidas realizadas posteriormente. O teste $t$ nãopareado foi aplicado para analisar as diferenças entre a PImáx e PEmáx entre os grupos. Foi aplicado o teste de correlação de Pearson para analisar associações entre as medidas de PI e PE com as diferentes cargas utilizadas.

\section{Resultados}

A amostra do estudo foi composta inicialmente por 18 pacientes, no entanto ocorreram perdas amostrais: três crianças do Grupo A desistiram de participar do estudo e uma, desse mesmo grupo, foi a óbito, resultando uma amostra final de 14 pacientes: cinco no Grupo A e nove no Grupo B. Dentre os sujeitos que compuseram a amostra total, cinco eram do sexo masculino e nove do sexo feminino, com média de idade de $8,3 \pm 2,6$ anos. Ao avaliar a faixa etária nos grupos, foi possível observar que os participantes apresentaram média de 7,0 22,91 e 9,0 2,5 anos nos Grupos $\mathrm{A}$ e $\mathrm{B}$, respectivamente.

A análise das variáveis mensuradas na avaliação inicial evidenciou que não houve diferença estatisticamente significativa entre os Grupos A e B, como observado na Tabela 1. A avaliação da força dos músculos respiratórios ao término de dez semanas constatou que, no Grupo A, as variáveis PImáx e PEmáx apresentaram diferença significativa entre a avaliação inicial e final do treinamento muscular inspiratório (Tabela 2). Para a PImáx, o cálculo de coeficiente d de Cohen foi de 1,01, com um incremento de $35 \%$ e, para a PEmáx, o cálculo foi de 1,18 , com aumento de $36 \%$ entre as avaliações, considerados como um tamanho de efeito "grande" e "muito grande", respectivamente ${ }^{(24)}$. No Grupo $B$, não foi observada diferença entre a avaliação inicial e a final, como demonstra a Tabela 2. Além disso, não foi observada diferença significativa entre os Grupos A e B ao final do estudo ( $p=0,541$ e $p=0,996$ para PImáx e PEmáx, respectivamente).

O Gráfico 1 demonstra a correlação semanal entre os níveis de carga utilizada e a PImáx. Esta se mostrou forte e positiva, obtendo significância estatística $(\mathrm{r}=0,9292 ; p<0,0001)$. No estudo da correlação da carga utilizada com a PEmáx também foi encontrada correlação positiva significativa $(\mathrm{r}=0,7191 ; p=0,0001)$.

Tabela 1 - Características respiratórias das crianças com leucemia aguda dos Grupos A e B na avaliação inicial, descritas em média \pm desvio padrão

\begin{tabular}{lccc}
\hline Variáveis & Grupo A & Grupo B & Valor de $\boldsymbol{p}$ \\
\hline IMC $\left(\mathrm{kg} / \mathrm{cm}^{2}\right)$ & $15,9 \pm 0,6$ & $17,3 \pm 2,8$ & 0,287 \\
Expansão axilar $(\mathrm{cm})$ & $3,7 \pm 1,4$ & $4,5 \pm 1,3$ & 0,285 \\
Retração axilar $(\mathrm{cm})$ & $3,9 \pm 1,3$ & $4,6 \pm 1,2$ & 0,313 \\
Expansão xifoide $(\mathrm{cm})$ & $3,5 \pm 2,0$ & $4,0 \pm 1,3$ & 0,535 \\
Retração xifoide $(\mathrm{cm})$ & $3,4 \pm 2,0$ & $4,0 \pm 1,3$ & 0,467 \\
PI inicial $\left(\mathrm{cmH}_{2} \mathrm{O}\right)$ & $48,8 \pm 15,9$ & $57,7 \pm 8,1$ & 0,185 \\
PE inicial $\left(\mathrm{cmH}_{2} \mathrm{O}\right)$ & $61,4 \pm 19,0$ & $78,5 \pm 16,9$ & 0,106 \\
\hline
\end{tabular}

IMC: índice de massa corpórea; PI: pressão inspiratória; PE: pressão expiratória.

Tabela 2 - Força dos músculos respiratórios (PI e PE) dos Grupos A e B durante avaliação inicial (A1) e após dez semanas (A2), em média \pm desvio padrão

\begin{tabular}{llccccc}
\hline \multirow{2}{*}{ Variáveis } & \multicolumn{2}{c}{$\mathrm{PI}\left(\mathrm{cmH}_{\mathbf{2}} \mathbf{O}\right)$} & \multicolumn{4}{c}{$\mathrm{PE}\left(\mathrm{cmH}_{\mathbf{2}} \mathbf{O}\right)$} \\
\cline { 2 - 6 } & $\mathbf{A 1}$ & $\mathbf{A 2}$ & Valor de $\boldsymbol{p}$ & $\mathbf{A 1}$ & $\mathbf{A 2}$ & Valor de $\boldsymbol{p}$ \\
\hline Grupo A & $48,8 \pm 15,9$ & $66,0 \pm 21,6$ & 0,008 & $61,4 \pm 19,0$ & $83,4 \pm 22,4$ & 0,044 \\
Grupo B & $57,7 \pm 8,1$ & $59,8 \pm 15,4$ & 0,623 & $78,5 \pm 16,9$ & $83,4 \pm 11,0$ & 0,350 \\
\hline
\end{tabular}

PI: pressão inspiratória; PE: pressão expiratória. 
Em adição, compararam-se os valores encontrados para PImáx e PEmáx na avaliação inicial das crianças participantes com idade a partir de sete anos e os valores de referência propostos por Wilson et al ${ }^{(25)}$. Foi possível identificar que 80 e $70 \%$ das crianças apresentavam redução da PImáx e PEmáx respectivamente.

\section{Discussão}

O elevado percentual observado neste estudo de crianças com valores de PImáx e PEmáx abaixo do previsto para a normalidade sugere que a avaliação dos músculos respiratórios pode ser essencial para o manejo clínico dos pacientes com leucemia aguda. Essa informação é reforçada pela literatura no tocante às alterações metabólicas encontradas nos pacientes com câncer atreladas aos efeitos colaterais produzidos pelo tratamento tradicional, promovendo um ciclo vicioso de perda de massa muscular, diminuição nos níveis de atividade física, resultando em aumento nos níveis de fadiga e fraqueza generalizada ${ }^{(4)}$.

O incremento observado na força respiratória no grupo de crianças que realizou TMI indica que a proposta deste estudo utilizou critérios apropriados. Já são bem documentados na literatura os efeitos do treinamento dos músculos respiratórios, relatados a partir de 1976 por Leith e Bradley ${ }^{(12)}$ ao estudarem adultos saudáveis. No estudo clássico, esses autores sugeriram que os músculos respiratórios exibem adaptação similar ao treinamento realizado em outros grupos musculares e evidenciaram um aumento em torno de $55 \%$ na força muscular inspiratória nos sujeitos submetidos ao treinamento.Entretanto, a inexistência de um consenso na literatura acerca de critérios ideais para a recuperação da força dos músculos respiratórios tem sido uma grande limitação para o desenvolvimento de novos protocolos. Esse aspecto é ainda mais relevante quando a população é composta por crianças. Poucos estudos foram desenvolvidos para essa faixa etária e, geralmente, estão associados a pesquisas com pacientes que apresentam quadro clínico de obstrução brônquica, frequentemente observados na asma e na fibrose cística ${ }^{(16,17)}$.

Apesar de a leucemia representar de 25 a $35 \%$ dentre os tipos de câncer diagnosticados na infância( ${ }^{(3)}$, suas repercussões clínicas, bem como nos músculos respiratórios, necessitam ser melhor investigadas para fornecer as informações necessárias para uma intervenção adequada. Essa limitação se estende desde a carência dos valores de referência para as pressões respiratórias máximas na faixa etária de maior

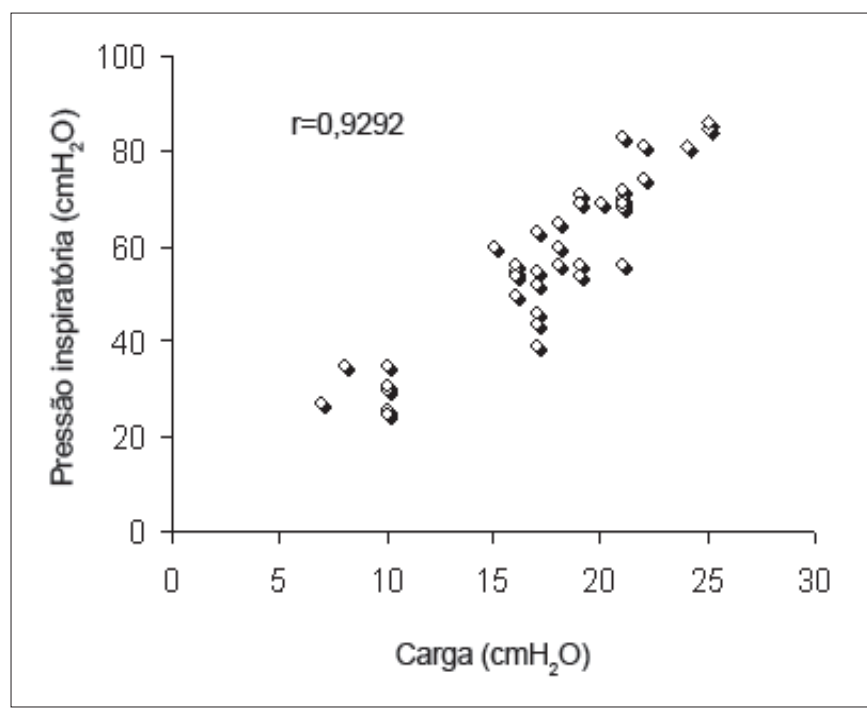

Gráfico 1 - Correlação da pressão inspiratória máxima (PImáx) com a carga.

acometimento da leucemia aguda, abaixo de cinco anos ${ }^{(3)}$, e à inexistência de protocolos para treinar a musculatura respiratória nessa população. Barsevick et $a^{(26)}$ relataram haver forte evidência do benefício do exercício aos pacientes que relatam fadiga, porém até o presente momento inexistem estudos que realizem TMI em crianças com leucemia aguda.

Os achados deste estudo indicam que a magnitude da carga adotada (30\% da PImáx) e o tempo de treinamento foram adequados e bem tolerados pelas crianças estudadas. Um estudo realizado com crianças asmáticas que utilizaram carga superior ( $40 \%$ da PImáx) e treinamento de menor duração (sete semanas) demonstrou melhora significativa da força muscular inspiratória no grupo treinado em relação aos controles ${ }^{(16)}$. Outros estudos disponíveis na literatura alcançaram incremento na força dos músculos inspiratórios em diversas populações (doença pulmonar obstrutiva crônica, asma, fibrose cística, doenças neuromusculares, insuficiência cardíaca congestiva, bronquiectasia e cirurgias toracoabdominais) e utilizaram cargas que variaram de 20 a $60 \%$ da PImáx e duração de treinamento entre quatro e 12 semanas $^{(13-17,19,21,27)}$.

Embora a proposta de treinamento tenha sido direcionada ao incremento da força muscular inspiratória das crianças avaliadas no Grupo A, foi evidenciado ganho real das PEmáx. Estudos prévios reforçam os achados do presente estudo, mostrando aumento da PEmáx nos grupos que realizaram o TMI ${ }^{(13,16,21)}$. Lima et $a^{(16)}$ sugeriram que a própria ação mecânica aumentada dos músculos inspiratórios, incluindo os músculos intercostais externos que têm comprovada 
participação acessória na expiração, proporcionou maior mobilidade toracoabdominal e consequente reorganização mecânica de todos os músculos envolvidos na respiração.

A inexistência de diferença entre os grupos avaliados ao comparar as pressões respiratórias máximas ao término do estudo também foi observada por outros autores ${ }^{(13,28)}$. No atual estudo, esse resultado pode decorrer do fato de todas as perdas amostrais terem ocorrido em um único grupo e pelo fato de as crianças controle apresentarem maior média de índice de massa corpórea (IMC) e idade. Estudos prévios com o propósito de estabelecer, para diversas populações, equações preditivas e valores de normalidade para pressões respiratórias máximas já indicavam que a força dos músculos respiratórios pode sofrer variabilidade dependendo de aspectos como sexo, idade, estatura e peso ${ }^{(22,25,29)}$.

Alguns pontos foram considerados como limitação deste estudo, tais como as perdas amostrais ocorridas, a impossibilidade momentânea de realizar um estudo multicêntrico e a não-realização de um treinamento placebo no

\section{Referências bibliográficas}

1. Elman I, Silva MEMP. Acute lymphocytic leukemia in children: analysis of detection thresholds for basic tastes according to gender. Rev Bras Cancerol 2007;53:297-303.

2. Belson M, Kingsley B, Holmes A. Risk factors for acute leukemia in children: a review. Environ Health Perspect 2007;115:138-45.

3. Reis RS, Santos MO, Thuler LC. Incidência de tumores pediátricos no Brasil. Rev Bras Cancerol 2007;53:5-15.

4. Battaglini CL, Bottaro M, Campbell JS, Novaes J, Simão R. Atividade física e níveis de fadiga em pacientes portadores de câncer. Rev Bras Med Esporte 2004; 10:98-104.

5. Dimeo FC. Effects of exercise on cancer-related fatigue. Cancer 2001; 92:1689-93.

6. Ryan JL, Carroll JK, Ryan EP, Mustian KM, Fiscella K, Morrow GR. Mechanisms of cancer-related fatigue. Oncologist 2007;12:22-34.

7. Hofman M, Ryan JL, Figueroa-Moseley CD, Jean-Pierre P, Morrow GR. Cancer-related fatigue: the scale of the problem. Oncologist 2007; 12:4-10.

8. Curt GA, Breitbart W, Cella D, Groopman JE, Horning SJ, Itri LM et al. Impact of cancer-related fatigue on the lives of patients: new findings from the Fatigue Coalition. Oncologist 2000;5:353-60.

9. Marcucci FCl. Physiotherapy on palliative care with cancer patients. Rev Bras Cancerol 2005;51:67-77.

10. Halarem BL. Relação da reabilitação com a inatividade. In: Kottke FJ, Lehmann JF. Tratado de medicina física e reabilitação de Krusen. $4^{\text {a }}$ ed. São Paulo: Manole; 1994. p. 1105-24.

11. Wolfson MR, Shaffer TH. Musculatura respiratória: fisiologia, avaliação e tratamento. In: Irwin S, Tecklin JS. Fisioterapia cardiopulmonar. $3^{\mathrm{a}}$ ed. São Paulo: Manole; 2003. p. 318-33.

12. Leith $D E$, Bradley M. Ventilatory muscle strenght and endurance training. J Appl Physiol 1976;41:508-16.

13. Klefbeck B, Nedjad JH. Effect of inspiratory muscle training in patients with multiple sclerosis. Arch Phys Med Rehabil 2003;84:994-9.
Grupo Controle. Acredita-se ser necessária a continuidade do estudo em parceria com outros centros de referência, permitindo a análise de uma amostra mais representativa. Uma população de estudo mais representativa possibilitaria o pareamento de idade, sexo e IMC entre os grupos. Além disso, seria interessante a utilização de um meio lúdico, como música ou vídeo durante o treinamento, a fim de torná-lo mais atrativo para a criança, e a avaliação de outras variáveis como resistência muscular, tolerância ao exercício, dispneia e qualidade de vida.

A ausência de estudos que investiguem as repercussões da quimioterapia na diminuição da força dos músculos respiratórios em crianças com leucemia aguda associada aos achados preliminares deste estudo apontam para a necessidade de avaliação e intervenção com respeito aos músculos respiratórios e às repercussões consequentes à fraqueza muscular. No entanto, os resultados aqui apresentados apontam um caminho inicial ao disponibilizar um protocolo de TMI que possibilitou o incremento das pressões respiratórias máximas nas crianças estudadas.

14. di Mambro TR, Figueiredo PH, Wanderley TR, Kristki AL, Guimarães FS. Treinamento muscular inspiratório na doença pulmonar obstrutiva crônica: impacto na qualidade de vida, intolerância ao esforço e dispnéia. Fisioter Pesqui 2007;14:65-71.

15. Newall C, Stockley RA, Hill SL. Exercise training and inspiratory muscle training in patients with bronchiectasis. Thorax 2005;60:943-8.

16. Lima EV, Lima WL, Nobre A, Santos AM, Brito LM, Costa MR. Treinamento muscular inspiratório e exercícios respiratórios em crianças asmáticas. J Bras Pneumol 2008;34:552-8.

17. Galvão F. Avaliação dos efeitos do treinamento muscular inspiratório em pacientes com fibrose cística através do uso do threshold IMT [dissertação]. Campinas: Universidade Estadual de Campinas; 2006.

18. Budweiser S, Moertl M, Jörres R, Windisch W, Heinemann F, Pfeifer M. Respiratory muscle training in restrictive thoracic disease: a randomized controlled trial. Arch Phys Med Rehabil 2006;87:1559-65.

19. Arcêncio L, Souza MD, Bortolin BS, Fernandes AC, Rodrigues AJ, Evora PR. Cuidados pré e pós-operatórios em cirurgia cardiotorácica: uma abordagem fisioterapêutica. Rev Bras Cir Cardiovasc 2008;23:400-10.

20. Burnetto AF, Paulin E, Yamaguti WP. Comparação entre a escala de Borg modificada e a escala de Borg modificada análogo visual aplicadas em pacientes com dispnéia. Rev Bras Fisioter 2002;6:41-5.

21. Fregonezi GA, Resqueti VR, Güell R, Pradas J, Casan P. Effects of 8-week, interval-based inspiratory muscle training and breathing retraining in patients with generalized myasthenia gravis. Chest 2005;128: 1524-30.

22. Black LF, Hyatt RE. Maximal respiratory pressures: normal values and relationship to age and sex. Am Rev Respir Dis 1969;99:696-702.

23. Souza RB. Pressões respiratórias estáticas máximas. J Pneumol 2002;28:S155-65.

24. Thalheimer W, Cook S. Work-Learning Research, Inc. [homepage on the 
Internet]. How to calculate effect sizes from published research articles: a simplified methodology [cited 2002 Aug]. Available from: http://work-learning. com/effect_sizes.htm

25. Wilson SH, Cooke NT, Edwards RH, Spiro SG. Predicted normal values for maximal respiratory pressures in Caucasian adults and children. Thorax 1984;39:535-8.

26. Barsevick AM, Newhall T, Brown S. Management of cancer-related fatigue. Clin J Oncol Nurs 2008;12:21-5.

27. Chiappa GR. Efeitos do treinamento muscular inspiratório em pacientes com insuficiência cardíaca: impacto na capacidade funcional, na oscilação da ventilação e na qualidade de vida [dissertação]. Porto Alegre: Universidade Federal do Rio Grande do Sul; 2003.

28. Kunikoshita LN, Silva YP, Silva TL, Costa D, Jamani M. Efeitos de três programas de fisioterapia respiratória (PFR) em portadores de DPOC. Rev Bras Fisioter 2006; 10:449-55.

29. Neder JA, Andreoni S, Lerario MC, Nery LE. Reference values for lung function tests. II. Maximal respiratory pressures and voluntary ventilation. Braz J Med Biol Res 1999;32:719-27. 